\title{
Discovery and Optimization of Small Molecules Targeting the Protein-Protein Interaction of Heat Shock Protein 90 (Hsp90) and Cell Division Cycle 37 (Cdc37) As Orally Active Inhibitors for the treatment of colorectal cancer
}

\section{Supplementary Information}

Lei Wang 1,2, Jingsheng Jiang 1,2, Lixiao Zhang 1,2, Qiuyue Zhang 1,2, Jianrui Zhou 1,2, $\mathrm{Li} \mathrm{Li}^{1,2}$, Xiaoli Xu ${ }^{1,2, *}$, Qidong You ${ }^{1,2, *}$

${ }^{1}$ State Key Laboratory of Natural Medicines and Jiang Su Key Laboratory of Drug Design and Optimization, China Pharmaceutical University, Nanjing 210009, China.

${ }^{2}$ Department of Medicinal Chemistry, School of Pharmacy, China Pharmaceutical University, Nanjing 210009, China. 


\section{Contents}

1. Table S1. Determination of 17h, 18h, 19, 20, 21 and AT13387 of Hsp90 ATPase activity in vitro.

2. Table S2. The change of Gibbs free energy from computational calculation and ITC experiments of the compound 17h, 18h, 19, 20, 21 and DDO-5936.

3. Figure S1. Binding affinities determined by BLI assay.

4. Figure S2. Binding affinities determined by ITC assay.

5. Figure S3. CPMG spectrum of $18 \mathrm{~h}$ and $18 \mathrm{~h}$ with Hsp90 WT or mutants.

6. Figure S4. Calculation of the change of Gibbs free energy of DDO-5936 and 18h at different dynamic trajectory time point.

6. Figure S5. H\&E of organs in different groups of mice with compound $18 \mathrm{~h}$ and DDO5936.

8. Figure S6. Representative ${ }^{1} \mathrm{H}-\mathrm{NMR}$ spectrum, ${ }^{13} \mathrm{C}$ NMR spectrum and HPLC of $18 \mathrm{~h}$. 
Table S1. Determination of 17h, 18h, 19, 20, 21 and AT13387 of Hsp90 ATPase activity in vitro.

\begin{tabular}{|c|c|c|c|}
\hline Comp. & $\mathbf{I C}_{\mathbf{5 0}}(\boldsymbol{\mu M})$ & Comp. & $\mathbf{I C}_{\mathbf{5 0}}(\boldsymbol{\mu M})$ \\
\hline $\mathbf{1 7 h}$ & $>100$ & $\mathbf{2 0}$ & $>100$ \\
\hline $\mathbf{1 8 h}$ & $>100$ & $\mathbf{2 1}$ & $>100$ \\
\hline $\mathbf{1 9}$ & $>100$ & $\mathbf{A T 1 3 3 8 7}$ & $0.15 \pm 0.04$ \\
\hline
\end{tabular}


Table S2. The change of Gibbs free energy from computational calculation and ITC experiments of the compound 17h, 18h, 19, 20, 21 and DDO-5936.

\begin{tabular}{|c|c|c|}
\hline Compound. & $\boldsymbol{\Delta} \mathbf{G}_{\text {calculation }}(\mathbf{k c a l} / \mathbf{m o l})$ & $\boldsymbol{\Delta} \mathbf{G}_{\text {experiment }}(\mathbf{k c a l} / \mathbf{m o l})$ \\
\hline $\mathbf{1 7 h}$ & -12.74 & -7.86 \\
\hline $\mathbf{1 8 h}$ & -16.39 & -8.32 \\
\hline $\mathbf{1 9}$ & -10.35 & -7.34 \\
\hline $\mathbf{2 0}$ & -0.49 & NA \\
\hline $\mathbf{2 1}$ & -10.97 & -7.01 \\
\hline DDO-5936 & -13.24 & -7.14 \\
\hline
\end{tabular}



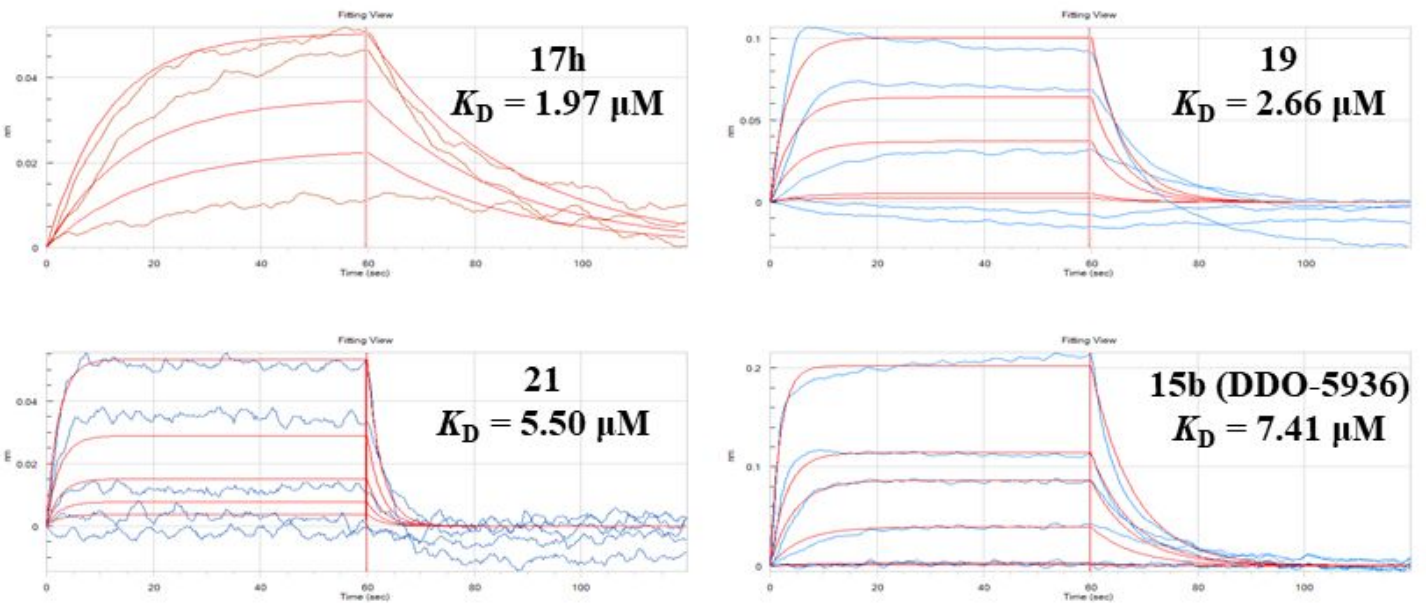

Figure S1. Binding affinities of 17h, 19, 21 and 15b (DDO-5936) determined by BLI assay. 


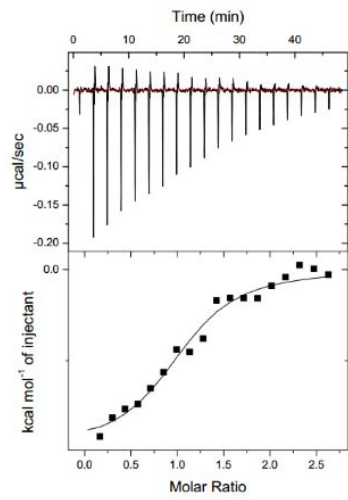

$17 \mathrm{~h}$ $K_{d}=1.73 \mu \mathrm{M}$

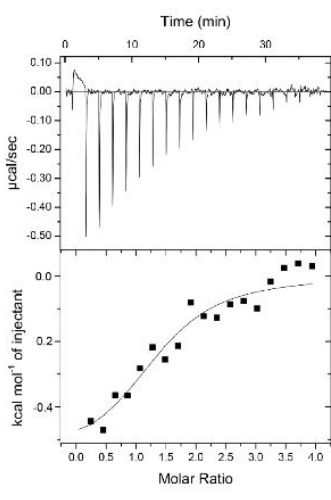

19

$K_{d}=4.17 \mu \mathrm{M}$

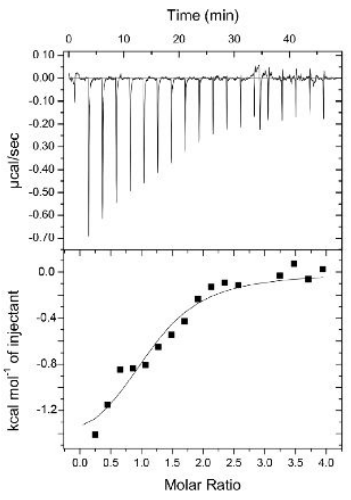

21

$K_{d}=\mathbf{2 . 4 0} \mu \mathrm{M}$

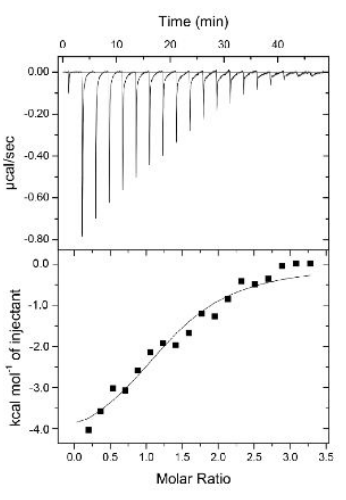

15b (DDO-5936)

$K_{d}=5.68 \mu \mathrm{M}$

Figure S2. Binding affinities of 17h, 19, 21 and 15b (DDO-5936) determined by ITC assay. 


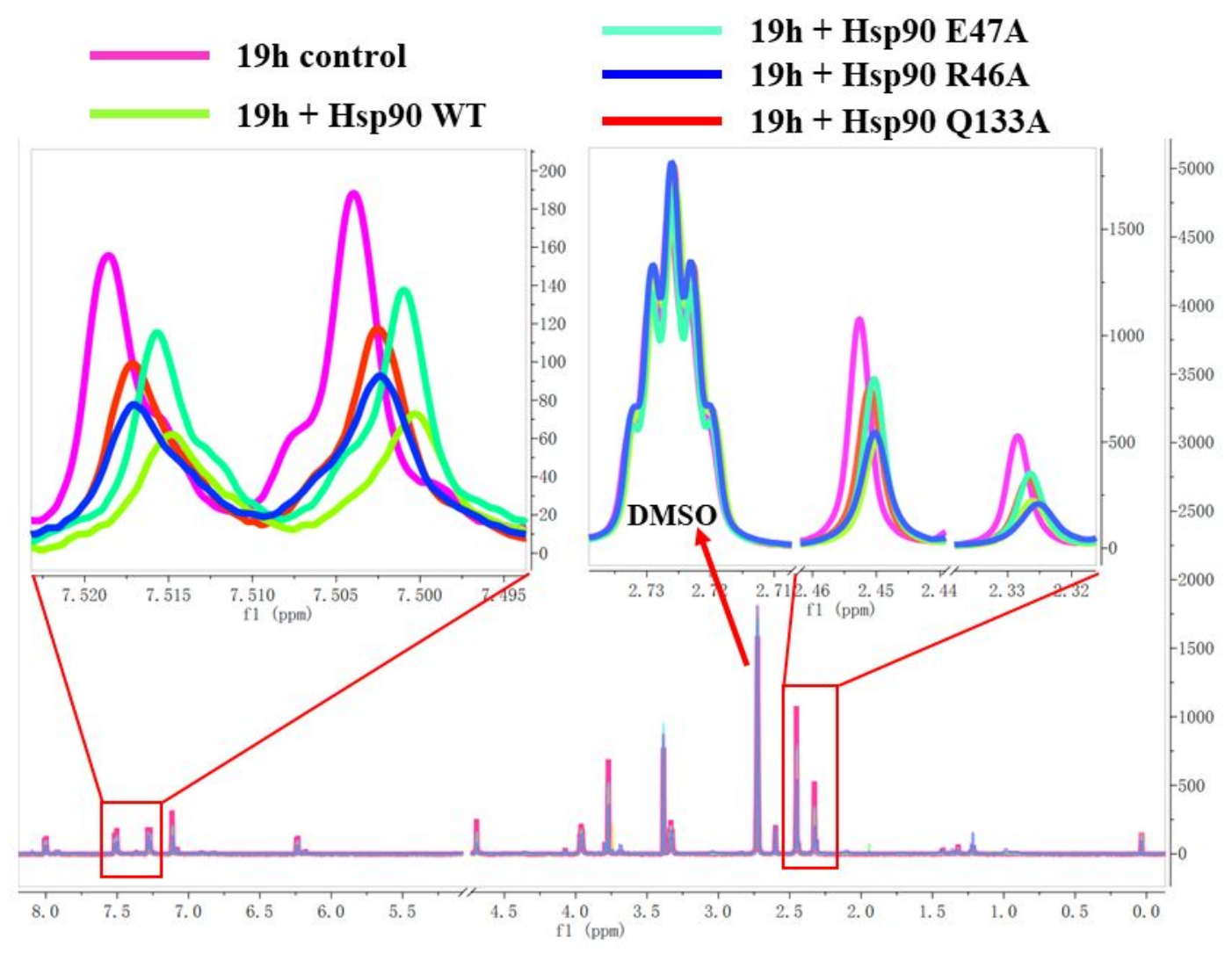

Figure S3. CPMG spectrum of $200 \mu \mathrm{M} 18 \mathrm{~h}$ (violet) with $20 \mu \mathrm{M}$ Hsp90 wild type (light green) and three mutants (20 $\mu$ M Hsp90 R46A, blue; $20 \mu \mathrm{M}$ Hsp90 E47A, green; $20 \mu M$ Hsp90 Q133A, red). 


\section{$\Delta \mathbf{G}$ calculated from dynamic trajectory}

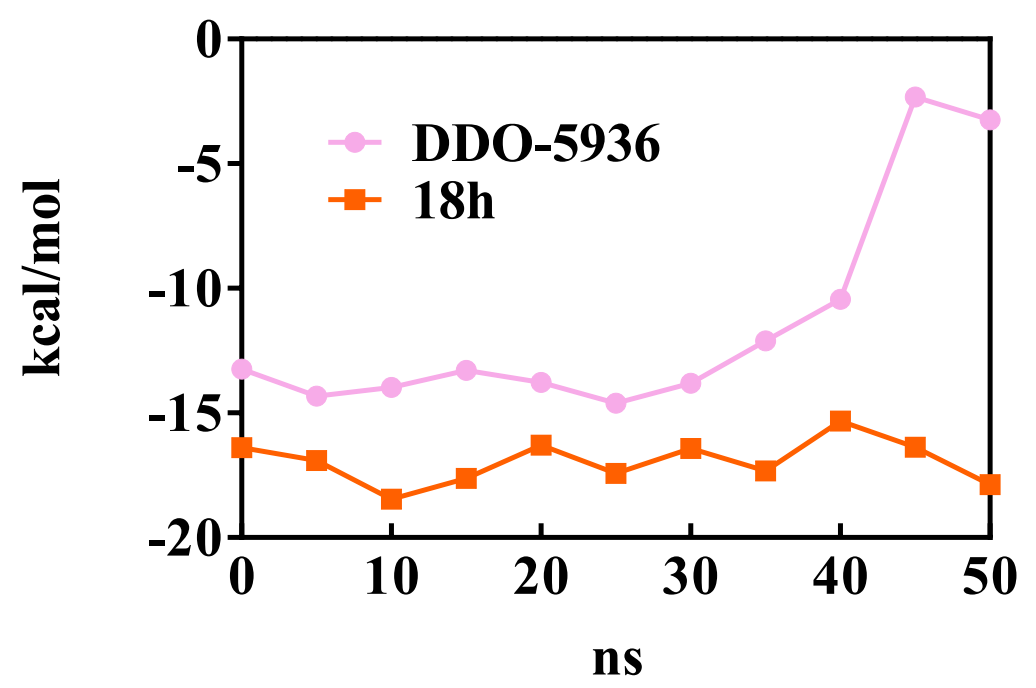

Figure S4. Calculation of the change of Gibbs free energy of DDO-5936 and $18 \mathrm{~h}$ at different dynamic trajectory time point. 


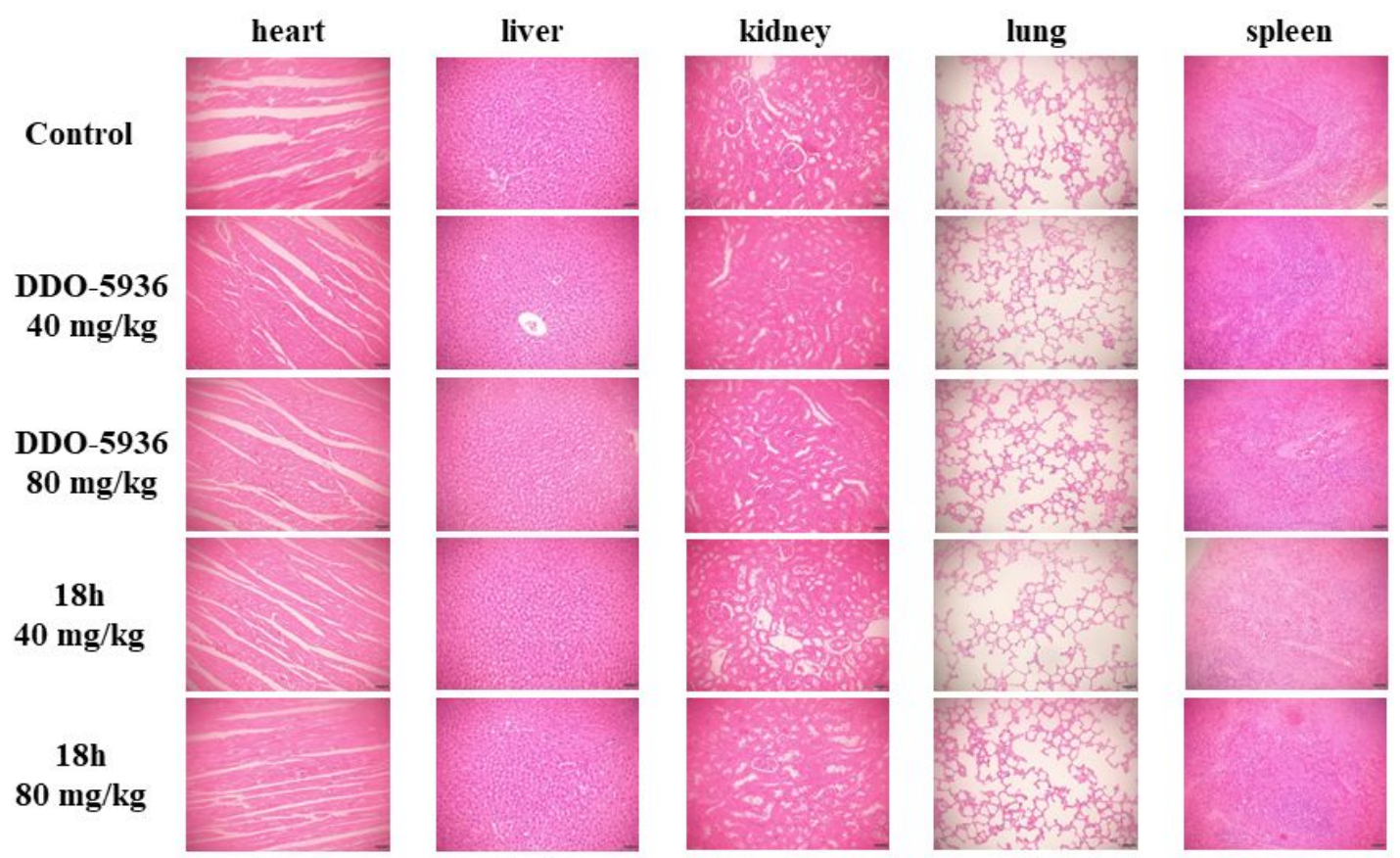

Figure S5. H\&E of organs in different groups of mice with compound $18 \mathrm{~h}$ and DDO-5936. Scale bars represent $50 \mu \mathrm{m}$. 

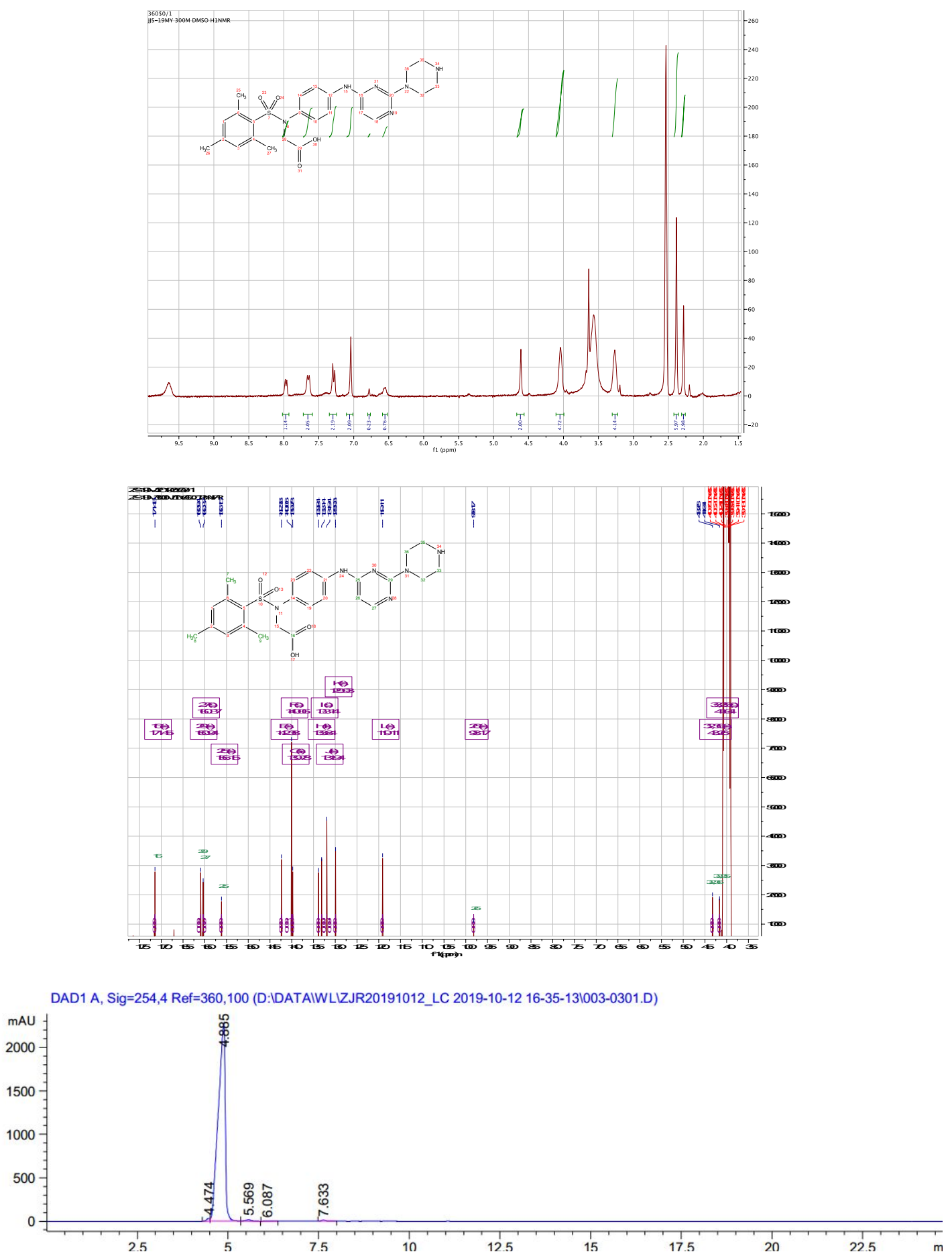

Figure S6. Representative ${ }^{1} \mathrm{H}-\mathrm{NMR}$ spectrum, ${ }^{13} \mathrm{C}$ NMR spectrum and HPLC of $18 \mathrm{~h}$. 